\title{
Special issue on critical considerations around eInnovations in health
}

\author{
D. Vogel $\cdot$ N. Wickramasinghe
}

Received: 18 February 2013 / Accepted: 5 March 2013 /Published online: 29 March 2013

(C) IUPESM and Springer-Verlag Berlin Heidelberg 2013

Today, it is indeed the best of times and the worst of times when it comes to healthcare delivery. On one hand healthcare globally is facing tremendous challenges to provide effective, efficient, efficacious patient centric care and on the other hand we are witnessing the most intense period of e-health solution development yet which holds the promise of realising a superior state for healthcare delivery. At such a time there are clearly several considerations that must be considered regarding the technology innovations in healthcare, or as we have coined the term eInnovations in health. In an attempt to highlight such key issues and developments we have compiled the following special issue. It was never our goal to provide an exhaustive list of topics but rather a pot-pourri of topics that focus on important aspects of eInnovations in health that will help to stimulate further debate and engage more research in this vital area.

The eight papers that make up this special issue include:

"Evaluation of Microsoft HealthVault and Google Health Personal Health Records" by Ali Sunyaev This paper examines and compares the design of application programming interfaces of two personal health record systems.

"Tailoring Software Process Capability/ Maturity Models for the Health Domain" by von Wangenheim et al. which examines web-based asynchronous store and forward telemedicine systems for diagnostic purposes.

\section{Vogel}

Information Systems Department,

City University of Hong Kong, 83 Tat Chee Avenue,

Kowloon Tong, Hong Kong

e-mail: isdoug@cityu.deu.hk

N. Wickramasinghe $(\bowtie)$

Epworth HealthCare \& RMIT University,

GPO Box 2476, Melbourne, VIC 3001, Australia

e-mail: nilmini.work@gmail.com
"The Influence of EHR Components on Admission Decisions" by Assuli et al. which looks at an ED environment to assess the specific data components that improve the process of decision making with regard to electronic health record design.

"Toward a Typology of Health 2.0 Collaboration Platforms and Websites" Kordzadeh and Warren This paper develops a typology for Health 2.0 collaboration platforms and websites and examines these keeping in mind the 2 major actors: the providers and the consumers

"A Rule-Based Transformation System for Converting Semi-Structured Medical Documents" by Heurix et al. which focuses on the design and development of a rule based transformation system that converts semi-structured texts into standardised formats.

"Some promising areas for IS research in the hospital industry: implications from a case study of operating room scheduling" by $\mathrm{He}$ et al. which serves to examine issues with operating room scheduling at a surgery department in the Midwest, US.

"A Phased Approach to Implementing Radio Frequency Identification Technologies within the Perioperative Process" by Ryan et al. which looks at the critical considerations and technology issues relating to implementing RFID technology into a hospital context.

And finally "Designing Healthy Living Support; Mobile applications added to hybrid (e)Coach Solution" by Simons et al.

We hope our readers enjoy this special issue and it serves to stimulate and foster more research in this growing and key area.

The Special Issue editors

Doug and Nilmini

Feb 2013 